\title{
Maturação e qualidade fisiológica das sementes de cumbaru em função do período de
}

\section{coleta dos frutos}

Maturation and physiological quality of cumbaru seeds for the period of collection of fruits

Madurez y calidad fisiológica de las semillas de cumbaru según el período de recolección de los

Jeyson Conceição Nascimento ORCID: https://orcid.org/0000-0002-2214-1926 Universidade do Estado de Mato Grosso, Brasil

E-mail: jeysonnascimento1@ hotmail.com

Marcella Karoline Cardoso Vilarinho ORCID: https://orcid.org/0000-0003-1410-142X Universidade do Estado de Mato Grosso, Brasil

E-mail: marcella.vilarinho@unemat.br

Daniela Soares Alves Caldeira ORCID: https://orcid.org/0000-0002-4345-3718 Universidade do Estado de Mato Grosso, Brasil

E-mail: danielacaldeira@unemat.br

Luis Augusto Magalhães Antoniacomi ORCID: https://orcid.org/0000-0001-5462-1118 Universidade do Estado de Mato Grosso, Brasil E-mail: luisantoniacomi@hotmail.com

Altacis Junior de Oliveira

ORCID: https://orcid.org/0000-0002-6787-7160 Universidade do Estado de Mato Grosso, Brasil

E-mail: altacismarquesfig@ @otmail.com

Taniele Carvalho de Oliveira

ORCID: https://orcid.org/0000-0002-6900-6449 Universidade do Estado de Mato Grosso, Brasil E-mail: tani.ele@ hotmail.com

Gustavo Ferreira da Silva ORCID: https://orcid.org/0000-0002-6673-6446 Universidade Estadual Paulista "Júlio de Mesquita Filho", Brasil

E-mail: ferreirasilvagustavo@gmail.com

Andrea dos Santos Oliveira

ORCID: https://orcid.org/0000-0002-9061-2304 Universidade do Estado de Mato Grosso, Brasil

E-mail: andrea.santos.oliveira@ unemat.br

Marco Antonio Aparecido Barelli ORCID: https://orcid.org/0000-0002-6385-6733 Universidade do Estado de Mato Grosso, Brasil E-mail: mbarelli@unemat.br

Petterson Baptista da Luz ORCID: https://orcid.org/0000-0003-4067-0087 Universidade do Estado de Mato Grosso, Brasil E-mail: petterson@unemat.br

\section{Resumo}

O cumbaru também conhecido em algumas regiões como baru, cumbaru, barujo, coco-feijão ou cumaru é uma espécie amplamente distribuída no país. A madeira é de alta densidade e durável, e tanto a polpa do fruto, quanto as sementes são comestíveis, atraindo uma fauna diversificada de mamíferos e de insetos. Devido à grande exploração de sua madeira e principalmente por ser uma fonte de alimento, o fruto do cumbaru está cada vez mais difícil de ser encontrado. Com isso, objetivou-se com o presente trabalho estabelecer o período ideal de colheita através da maturação fisiológica de sementes de cumbaru, por meio da fisiologia e biometria de frutos e sementes. Os frutos foram coletados nas árvores, 180, 225 e 275 dias após a antese (DAA). O delineamento experimental utilizado foi o inteiramente casualisado em esquema fatorial 3x3 (três lotes e três períodos de coleta) com quatro repetições, sendo considerados 40 frutos por repetição. Foram avaliadas características morfológicas de frutos e sementes como (comprimento longitudinal, largura, espessura, peso fresco e volume) e características físicas e fisiológicas das sementes como: teor de água, germinação, primeira contagem de germinação, índice de velocidade de germinação e 
peso de mil sementes. Observou-se, ao longo dos períodos de coleta, valores crescentes para as variáveis de frutos e sementes, a melhor qualidade fisiológica deu-se nos $2^{\circ}$ e $3^{\circ}$ período de coleta.

Palavras-chave: Biometria; Dipteryx alata; Vog; Morfofisiologia.

\begin{abstract}
The cumbaru also known in some regions as baru, cumbaru, barujo, coco-beans or cumaru is a species widely distributed in the country. The wood is high density and durable, and both the fruit pulp and seeds are edible, attracting a diverse fauna of mammals and insects. Due to the great exploitation of its wood and mainly because it is a source of food, the fruit of cumbaru is increasingly difficult to find. With this, the aim of this work was to establish the ideal harvest period through the physiological maturation of cumbaru seeds, through the physiology and biometry of fruits and seeds. The fruits were collected from the trees, 180, 225 and 275 days after the anthesis (DAA). The experimental design used was the one entirely randomized in a $3 \times 3$ factorial scheme (three lots and three collection periods) with four repetitions, being considered 40 fruits per repetition. Morphological characteristics of the fruits and seeds were evaluated (longitudinal length, width, thickness, fresh weight and volume) and physical and physiological characteristics of the seeds such as: water content, germination, first germination count, germination speed index and weight of one thousand seeds. It was observed, along the collection periods, increasing values for the fruit and seed variables, the best physiological quality was observed during the 2 nd and 3rd collection periods.
\end{abstract}

Key words: Biometry; Dipteryx alata; Vog; Morphology.

\title{
Resumen
}

El cumbaru, también conocido en algunas regiones como baru, cumbaru, barujo, coco o cumaru, es una especie de amplia distribución en el país. La madera es de alta densidad y duradera, y tanto la pulpa de la fruta como las semillas son comestibles, atrayendo una fauna diversa de mamíferos e insectos. Debido a la gran explotación de su madera y principalmente porque es una fuente de alimento, el fruto del cumbaru es cada vez más difícil de encontrar. Este trabajo tenía como objetivo establecer el período de cosecha ideal a través de la maduración fisiológica de las semillas de cumbaru, a través de la fisiología y la biometría de los frutos y las semillas. Los frutos se recogieron de los árboles 180, 225 y 275 días después de la antesis (DAA). El diseño experimental utilizado fue totalmente aleatorio en un esquema factorial 3x3 (tres lotes y tres períodos de recolección) con cuatro repeticiones, considerándose 40 frutos por repetición. Se evaluaron las características morfológicas de los frutos y las semillas (longitud longitudinal, anchura, grosor, peso y volumen en fresco) y las características físicas y fisiológicas de las semillas como: contenido de agua, germinación, recuento de la primera germinación, índice de velocidad de germinación y peso de mil semillas. Se observó, a lo largo de los períodos de recolección, un aumento de los valores de las variables de frutos y semillas, la mejor calidad fisiológica se observó durante el segundo y tercer período de recolección.

Palabras clave: Biometría; Dipteryx alata; Vog; Morfofisiología.

\section{Introdução}

As frutíferas do Cerrado destacam-se por seu potencial agrícola devido à grande produção de frutos, entre elas a espécie Dipteryx alata Vog., uma árvore neotropical nativa do bioma Cerrado brasileiro que é o segundo maior ecossistema brasileiro e uma das maiores biodiversidades do mundo. Essa espécie é comumente conhecida como cumbaru, sendo também conhecido também em algumas regiões como baru, barujo, coco-feijão ou cumaru (Sano et al., 2014, Guimarães et al., 2019, Oliveira-Alves et al., 2020).

A madeira é de alta densidade e durável, e tanto a polpa do fruto, quanto as sementes são comestíveis, atraindo uma fauna diversificada de mamíferos e de insetos. Os bovinos também se alimentam dos frutos e das folhas, principalmente na época da estiagem. Sua casca e folhas são utilizadas na medicina popular e com grande potencial na indústria fitoterápica, já o consumo das sementes reduz a adiposidade e melhora o perfil lipídico em mulheres com sobrepeso e obesidade (Alvarenga et al., 2008, Souza et al., 2018).

A preocupação com relação à qualidade ambiental tem sido frequente, por isso, a produção de mudas de espécies florestais para recuperação de áreas degradadas e para uso extrativista, tem aumentado a demanda de materiais com boa qualidade morfofisiológica, o cumbaru é considerado uma espécie chave para o Cerrado, possui alta taxa de germinação e estabelecimento de mudas, sendo assim, promissora na recuperação de reservas legais e áreas de proteção permanentes (Sano et al., 2004, Silva, 2017). E para que os plantios com cumbaru se tornem mais produtivos e uniformes, faz-se necessária à 
utilização de sementes que provenham de frutos com procedência adequada e que apresentem boa uniformidade de germinação.

Devido a grande exploração de sua madeira para obtenção de carvão vegetal, e principalmente, por ser uma fonte de alimento de diferentes grupos de animais, o fruto do cumbaru está cada vez mais difícil de ser encontrado, isto porque estes são coletados quando caem ao chão, geralmente no período de julho a outubro, dificultando a obtenção do mesmo em grandes quantidades e uniformidade (Lorenzi, 1998; Oliveira \& Sigrist, 2008). Segundo os mesmos autores, é nesse período também, que as sementes apresentam maior potencial de germinação, coincidindo com a sua maturidade fisiológica.

Para ter sucesso no cultivo de espécies nativas, são necessárias informações sobre sua propagação. Porém, existem poucas recomendações relacionadas à produção de mudas de barueiro (Pinho et al., 2018). Nesse contexto, a qualidade de sementes de espécies florestais pode ser afetada por diversos fatores, como a ocorrência de ataque de insetos e doenças, a formação de sementes vazias ou embriões malformados e a deterioração decorrente de atrasos na colheita (Gomes et al., 2014, Silva et al., 2018).

Outro aspecto que reflete diretamente à qualidade das sementes é o período de sua coleta, que pode ser acompanhado através do desenvolvimento dos frutos, por meio das suas características morfofisiológicas. Observar período ideal de coleta dos frutos se faz importante uma vez que a velocidade de maturação dos frutos pode variar entre árvores de uma mesma espécie, de acordo com o local de coleta ou conforme o ano (Carvalho \& Nakagawa, 2000).

A fase em que as sementes apresentam máxima qualidade coincide com o ponto de maturação fisiológica, esse processo é constituído por uma série de alterações morfológicas, fisiológicas, funcionais e bioquímicas (aumento de tamanho, variações no teor de água, vigor e acúmulo de massa seca), que ocorrem a partir da fecundação e prosseguem até o momento em que as sementes atinjam a maturidade fisiológica (Hehenberger et al., 2012, Marcos Filho, 2015).

Um aspecto a ser observado para o acompanhamento da maturidade fisiológica das sementes é a observação das mudanças físicas externas dos frutos, como por exemplo, seu tamanho. Estudos realizados com outras espécies da região do Cerrado apontam que o estádio de maturação e o tamanho dos frutos influenciam diretamente na germinação e no vigor das sementes, contribuindo para a desuniformidade dos lotes.

Uma das formas de se detectar a viabilidade de um lote de frutos ou de sementes, e com isso conseguir um uso racional e mais eficaz desses materiais, é através da avaliação biométrica. O estudo da biometria constituiu uma importante ferramenta para a identificação da variabilidade genética em populações de uma mesma espécie, servindo como subsídio para análises de qualidade fisiológica das sementes, e suas relações aos fatores ambientais em que elas estão inseridas. (Gusmão et al., 2006; Zuffo et al., 2014).

Diversas pesquisas envolvendo a diversidade morfológica de frutos e sementes têm sido realizadas com o cumbaru. Soares et al., (2008), descrevem a distribuição espacial da variabilidade genética intra populacional de cumbaru. Já Arakaki et al. (2009), avaliando a alternativa de sustentabilidade em área de fragmento florestal do Cerrado, no Mato Grosso do Sul, utilizando cumbaru, observaram a ampliação da renda das famílias e melhoria da qualidade de vida, pela agregação de valor aos produtos oriundos do fruto dessa espécie.

Luz (2016) avaliou a variação genética entre e dentro de populações de Dipteryx alata vog. para caracteres morformétricos de plântulas, frutos e sementes, e observou-se diferenças significativas entre procedências e progênies para os caracteres dos frutos, sementes e plântulas, indicando a presença de variação genética. E Silva et al. (2020), estudou as alterações bioquímicas e fisiológicas em sementes de Dipteryx alata Vog. durante a germinação e envelhecimento acelerado, verificou que em conjunto, as alterações bioquímicas e fisiológicas podem ser utilizadas como marcadores da deterioração das sementes de D. alata induzida por altas temperaturas e umidade.

Diante do exposto, objetivou-se com o presente trabalho estabelecer o período ideal de colheita através da maturação 
fisiológica de sementes de cumbaru, por meio da fisiologia e biometria de frutos e sementes.

\section{Metodologia}

O estudo foi conduzido na Universidade do Estado de Mato Grosso, campus de Cáceres - MT. O clima da região segundo a classificação de Köppen é Aw, com estação seca de inverno e úmida no verão. A temperatura média anual é de 32,4 ${ }^{\circ} \mathrm{C}$, podendo atingir temperaturas de $40^{\circ} \mathrm{C}$ (Santos et al., 2009; Neves et al., 2011), e a pluviosidade média anual em torno de $1.370 \mathrm{~mm}$ (IBGE, 2015).

Os frutos de cumbaru (Dipteryx alata Vog.) foram obtidos de três matrizes localizadas em três regiões do município de Cáceres - MT, e colhidos ainda nas árvores, em três períodos após a antese (DAA) (180, 225 e 275 dias) tendo início em outubro de 2015 na safra de (2015/2016). Após a coleta, os frutos foram levados para o laboratório, acondicionados em caixas plásticas com aberturas laterais para circulação de ar. E por um período de duas semanas passaram por um processo de secagem ambiente, onde foram movimentados a cada dois dias para garantir a uniformização da retirada de água e também para evitar o surgimento de algum patógeno.

Foi utilizado o delineamento experimental inteiramente casualisado (DIC) em esquema fatorial simples 3x3 (três lotes e três períodos de coleta) com quatro repetições, sendo considerados 40 frutos por repetição.

Inicialmente, realizaram-se determinações biométricas do fruto: comprimento longitudinal (CLF), largura (LF) e espessura (EF), com o auxílio de um paquímetro digital; peso fresco do fruto (PFF) determinado em balança de precisão de 0,0001g e volume do fruto (VF) verificado a partir do volume de água deslocado após a imersão do fruto na proveta, contendo volume conhecido, conforme metodologia descrita por Basso (1999).

A remoção das sementes foi dada pelo rompimento do endocarpo dos frutos com auxílio de uma marreta. Logo após, determinou-se as seguintes avaliações biométricas: comprimento longitudinal (CLS), largura (LS) e espessura, (ES), feitas com auxílio de um paquímetro digital (mm), peso fresco da semente (PFS), determinado em balança de precisão de 0,0001g, e volume da semente (VS), obtido por meio dos valores de comprimento, largura e espessura (CLS x LS x ES) de cada uma.

Após as determinações biométricas, avaliou-se os seguintes parâmetros nas sementes:

a) Teor de água: realizado através do método da estufa a $105^{\circ} \mathrm{C} \pm 3^{\circ} \mathrm{C}$ por 24 horas (Brasil, 2009).

b) Teste de germinação: conduzido em câmara de germinação, com fotoperíodo de 12 horas, em temperatura de 25 ${ }^{\circ} \mathrm{C}$, com quatro repetições de 25 sementes por lote, sendo semeadas em bandejas plásticas contendo areia lavada e esterilizada, umedecida em $40 \%$ da capacidade de retenção de água. Antes da semeadura, as sementes foram desinfetadas com hipoclorito de sódio, $3 \%$ de cloro ativo, por três minutos. Feito isso, realizou-se a lavagem em água destilada (seis vezes), para a retirada de qualquer excesso (Botezelli et al., 2000). As contagens foram realizadas diariamente, computando-se as plântulas que emitiram o epicótilo. Os resultados foram expressos em porcentagem (Brasil, 2009).

c) Primeira contagem de germinação: Conduziu-se de acordo com a regra para análises de sementes, realizando-se leitura 7 dias após a semeadura (Brasil, 2009).

d) Índice de velocidade de germinação: Mediante contagem diária do número de plântulas germinadas até sua estabilização. O índice foi calculado de acordo com a fórmula proposta por Maguire (1962).

e) Peso de mil sementes: Conduziu-se de acordo com a regra para análises de sementes (Brasil, 2009).

Os dados de cada parâmetro das duas etapas foram submetidos à análise de variância, e as médias dos tratamentos pelo teste de Tukey a 5\% de probabilidade utilizando o programa estatístico SISVAR (Ferreira, 2014). 


\section{Resultados e Discussão}

Na Tabela 1 são apresentados valores médios de comprimento e largura referente à coleta aos 180, 225 e 275 dias após a antese (DAA). Observa-se que houve efeito entre a época de coleta e lotes, para o comprimento e largura dos frutos nos períodos avaliados. A colheita dos frutos aos 225 e 275 DAA apresentaram melhores resultados com relação ao comprimento no lote 1. Para a largura dos frutos, os maiores valores foram observados no lote 1 aos 275 DAA de coleta, sendo seus valores decrescentes nas coletas anteriores.

Tabela 1. Comprimento e largura dos frutos de cumbaru em diferentes épocas de colheita após antese.

\begin{tabular}{lcccccc}
\hline & \multicolumn{3}{c}{ Comprimento } & \multicolumn{3}{c}{ Largura } \\
\hline DAA & L1 & L2 & L3 & L1 & L2 & L3 \\
\hline 180 & $38,05 \mathrm{bA}$ & $38,85 \mathrm{bA}$ & $37,92 \mathrm{bA}$ & $30,87 \mathrm{cB}$ & $33,10 \mathrm{bA}$ & $31,83 \mathrm{bB}$ \\
225 & $43,24 \mathrm{aA}$ & $41,96 \mathrm{aB}$ & $38,25 \mathrm{bC}$ & $40,26 \mathrm{bA}$ & $34,03 \mathrm{abB}$ & $31,92 \mathrm{bC}$ \\
275 & $43,95 \mathrm{aA}$ & $41,95 \mathrm{aB}$ & $39,67 \mathrm{aC}$ & $41,67 \mathrm{aA}$ & $34,99 \mathrm{aB}$ & $33,36 \mathrm{aC}$ \\
\hline $\mathrm{Cv} \%$ & & 1,78 & & & 1,75
\end{tabular}

DAA= Dia após a antese. $\mathrm{L}=$ Lote. $\mathrm{L} 1=$ Lote $1 \mathrm{~L} 2=$ Lote $2 ; \mathrm{L} 3=$ Lote 3. Comprimento longitudinal do fruto (mm); largura do fruto (mm). Médias seguidas pela mesma letra, minúscula na coluna ou maiúscula na linha, não diferem entre si pelo teste de Tukey, ao nível de 5\% de probabilidade.

Fonte: Autores.

Os resultados do presente estudo concordam com Zuffo et al. (2014), que encontraram valores médios próximos aos encontrados na espécie, analisando a biometria dos frutos de Dipteryx alata Vog., onde os autores encontraram uma média geral das variáveis comprimento longitudinal do fruto e peso fresco nos três períodos de coleta, 41,28 mm e 11,73 g.

Os frutos de cumbaru apresentaram diferenças no comprimento, largura e espessura (Tabela 1 e 2), com acréscimos ao longo dos períodos de coleta. Para espessura dos frutos houve efeito do período de coleta, sendo observados aos 225 e 275 DAA resultados superiores aos 180 DAA (Tabela 2).

Tabela 2. Espessura dos frutos de cumbaru em diferentes épocas de colheita após antese.

\begin{tabular}{cc}
\hline DAA & Espessura \\
\hline 180 & $22,13 \mathrm{~b}$ \\
225 & $24,39 \mathrm{a}$ \\
275 & $25,99 \mathrm{a}$ \\
\hline $\mathrm{Cv} \%$ & 8,09 \\
\hline
\end{tabular}

$\mathrm{DAA}=$ Dia após a antese. Espessura (mm). Os valores seguidos pela mesma letra, na coluna, não diferem entre si pelo teste de Tukey (p< $0,05)$.

Fonte: Autores.

Uma possível explicação pode estar relacionada principalmente ao grau de maturidade dos frutos, que aumenta com o tempo em que ficam aderidas as plantas (225 e 275 DAA), ou até mesmo por características próprias das matrizes selecionadas como: a idade da planta, variabilidade genética e sanidade da matriz. De acordo com Ganga et al. (2009), a maioria das variações relacionadas aos frutos advém de variações fenotípicas existente entre as plantas e por componentes ambientais não controlados, como solo, clima, antropização, idade da planta, competição e a própria diferença genética entre os indivíduos.

Ao longo desse período de formação, os frutos ganharam massa, resultando em uma maior biometria nos períodos de tempo mais longos após a antese. Outra relação pode ser dada pelo tipo de solo da região, uma vez que as matrizes foram 
coletadas em pontos diferentes, e mesmo estando dentro de uma mesma região, a estrutura do solo, ausência ou presença de algum nutriente e a física do solo, podem interferir no desenvolvimento dos frutos.

Silva Jr. et al. (2012) descreveram que as diferenças encontradas entre os frutos podem estar relacionadas tanto às variações ambientais locais como estratégia na utilização de nutrientes e recurso hídrico disponível, como também à própria diversidade genotípica das populações, o que podem resultar em diferentes características fenotípicas para a espécie.

O volume e peso fresco de frutos foram modificados de acordo com a época de coleta e o lote (Tabela 3). Na medida em que se aumenta o período de coleta dos frutos ocorrem acréscimos no volume dos frutos, com melhores resultados aos 275 DAA.

Tabela 3. Volume e peso fresco dos frutos de cumbaru em diferentes épocas após antese.

\begin{tabular}{lcccccr}
\hline & \multicolumn{3}{c}{ Volume } & \multicolumn{3}{c}{ Peso Fresco } \\
\hline DAA & L1 & L2 & L3 & L1 & L2 & L3 \\
180 & $2,84 \mathrm{cA}$ & $1,82 \mathrm{cB}$ & $1,94 \mathrm{cB}$ & $8,57 \mathrm{cA}$ & $7,89 \mathrm{cA}$ & $8,62 \mathrm{bA}$ \\
225 & $3,79 \mathrm{bA}$ & $2,72 \mathrm{bB}$ & $2,27 \mathrm{bC}$ & $15,39 \mathrm{bA}$ & $11,08 \mathrm{bB}$ & $9,20 \mathrm{bC}$ \\
275 & $4,75 \mathrm{aA}$ & $3,65 \mathrm{aB}$ & $3,04 \mathrm{aC}$ & $20,05 \mathrm{aA}$ & $14,77 \mathrm{aB}$ & $12,69 \mathrm{aC}$ \\
\hline $\mathrm{Cv} \%$ & 4,98 & & & 5,47
\end{tabular}

DAA= Dia após a antese. $\mathrm{L}=$ Lote. $\mathrm{L} 1=$ Lote1; $\mathrm{L} 2=$ Lote $2 ; \mathrm{L} 3=$ Lote 3 . Volume do fruto $\left(\mathrm{cm}^{3}\right)$; Peso fresco (g). Médias seguidas pela mesma letra, minúscula na coluna ou maiúscula na linha, não diferem entre si pelo teste de Tukey, ao nível de 5\% de probabilidade.

Fonte: Autores.

Quanto ao volume e peso dos frutos, observa-se que as medias dos frutos cresceram conforme aumentaram os dias de coleta após o período de antese. Os resultados encontrados no presente estudo, corroboram aos encontrados por Zuffo et al. (2014), na qual essa variação entre volume e peso médio dos frutos pode estar relacionadas a fatores ambientais onde se localizam as matrizes.

De maneira geral, observa-se que as alterações nas características relacionadas aos frutos de cumbaru estão associadas aos períodos de coleta dos frutos, nota-se que a colheita ideal deve ocorrer aos 225 e 275 DAA. Sendo assim, a coleta quando realizada com frutos ainda nas árvores facilitam na seleção de sementes de qualidade, uma vez que há grande dificuldade na obtenção dos frutos maduros ao chão, principalmente pelos frutos serem palatáveis a uma diversidade de animais.

Os resultados do teor de água em sementes são expressos em porcentagem (base úmida) por lote estão apresentados na Tabela 4. Resultados encontrados por Ataíde et al. (2013) avaliando sementes de D. nigra, encontraram valores próximos de teores de água em dois lotes de sementes (7,92\% e 8,98\%), aos encontrados neste estudo. Esse baixo teor de água em sementes ortodoxas e oleaginosas (Aguiar et al., 2010), é considerado ideal permitindo manter as sementes viáveis por maior período. 
Tabela 4. Teor de água nas sementes de cumbaru em diferentes épocas após antese.

\begin{tabular}{cccc}
\hline & \multicolumn{3}{c}{ Teor de água (\%) } \\
\cline { 3 - 4 } & & 225 (DAA) & 275(DAA) \\
\hline L1 & 8,26 & 6,27 \\
L2 & 8,45 & 7,50 & 8,94 \\
\hline
\end{tabular}

Teores de água de sementes de cumbaru, obtidos pelo método estufa a $105^{\circ} \mathrm{C} \pm 3^{\circ} \mathrm{C}$ por 24 horas. L1 = Lote 1 ; L2 = Lote 2 ; L3 = Lote 3 . DAA= Dia após a antese.

Fonte: Autores.

Diversos aspectos ambientais são dispostos na literatura sobre o desenvolvimento das sementes, reflexos desses fatores são encontrados principalmente na biometria, peso e fisiologia das sementes. No entanto, a taxa de desenvolvimento das sementes é relativamente estável em diferentes ambientes, pois os ajustes no número de sementes produzidas pela planta ou pela comunidade vegetal podem manter um suprimento relativamente constante de assimilados para as mesmas (Marcos Filho, 2005).

Também foram observados efeitos entre período de coleta e lote estudado. Para o comprimento, sementes coletadas aos 225 e 275 DAA para os lotes 1 e 3 possuem resultados superiores aos 180 DAA. Já para o lote 2, a coleta realizada aos 275 DAA proporcionou sementes com maior comprimento. O mesmo comportamento foi verificado na largura e espessura das sementes (Tabela 5).

Tabela 5. Comprimento largura e espessura das sementes de cumbaru em diferentes épocas após antese.

\begin{tabular}{cccccccccc}
\hline & \multicolumn{3}{c}{ Comprimento } & \multicolumn{3}{c}{ Largura } & \multicolumn{3}{c}{ Espessura } \\
\hline & $\mathrm{L} 1$ & $\mathrm{~L} 2$ & $\mathrm{~L} 3$ & $\mathrm{~L} 1$ & $\mathrm{~L} 2$ & $\mathrm{~L} 3$ & $\mathrm{~L} 1$ & $\mathrm{~L} 2$ & $\mathrm{~L} 3$ \\
$\mathrm{P} 1$ & $0,0 \mathrm{bA}$ & $0,0 \mathrm{cA}$ & $0,0 \mathrm{bA}$ & $0,0 \mathrm{bA}$ & $0,0 \mathrm{cA}$ & $0,0 \mathrm{bA}$ & $0,0 \mathrm{bA}$ & $0,0 \mathrm{cA}$ & $0,0 \mathrm{bA}$ \\
$\mathrm{P} 2$ & $19,70 \mathrm{aA}$ & $17,57 \mathrm{bB}$ & $14,57 \mathrm{aB}$ & $9,08 \mathrm{aA}$ & $8,23 \mathrm{bA}$ & $6,71 \mathrm{aB}$ & $7,66 \mathrm{aA}$ & $6,10 \mathrm{bB}$ & $4,80 \mathrm{aC}$ \\
$\mathrm{P} 3$ & $18,13 \mathrm{aA}$ & $20,19 \mathrm{aA}$ & $14,59 \mathrm{aB}$ & $8,95 \mathrm{aA}$ & $9,28 \mathrm{aA}$ & $6,60 \mathrm{aB}$ & $7,59 \mathrm{aA}$ & $7,36 \mathrm{aA}$ & $4,42 \mathrm{aB}$ \\
\hline $\mathrm{Cv} \%$ & & 11,25 & & 8,98 & & & 9,53 & \\
\hline
\end{tabular}

DAA= Dia após a antese. $\mathrm{L}=$ Lote $. \mathrm{L} 1=$ Lote1 L2 = Lote 2; L3 = Lote 3. Comprimento longitudinal da semente (mm); Largura da semente $(\mathrm{mm})$; Espessura da semente $(\mathrm{mm})$. Médias seguidas pela mesma letra, minúscula na coluna ou maiúscula na linha, não diferem entre si pelo teste de Tukey, ao nível de $5 \%$ de probabilidade.

Fonte: Autores.

A biometria de sementes aos 225 e 275 DAA obtiveram maiores médias, quando comparados ao lote 1 . O que pode explicar os resultados obtidos é o fato de as características das sementes serem distintas entre as matrizes, em função de a espécie estudada ser uma espécie florestal não cultivada, que apresenta variações cíclicas.

As variações fenotípicas ou até mesmo irregularidade produtiva entre anos consecutivos pode estar associada a fatores genéticos e ambientais, como precipitação e temperatura (Felippi et al., 2012), podendo expressar modificações na morfologia, anatomia, taxa fotossintética, dentre outros fatores (Felippi et al., 2015). Desta maneira, o ambiente na sua formação e as características genéticas das matrizes originam diferenças significativas entre elas.

O volume e peso fresco das sementes foram afetados pela época de colheita de frutos e lotes (Tabela 6). Variações no volume foram observados, com melhores respostas aos 225 apenas no lote 1 e aos 275 DAA (lote 1 e 2). O peso Fresco das sementes destacou-se aos 275 DAA no lote 1. 
Research, Society and Development, v. 10, n. 1, e21610111589, 2021

(CC BY 4.0) | ISSN 2525-3409 | DOI: http://dx.doi.org/10.33448/rsd-v10i1.11589

Tabela 6. Volume e peso fresco das sementes de cumbaru em diferentes épocas após antese.

\begin{tabular}{lcccccc}
\hline & \multicolumn{3}{c}{ Volume $\left(\mathbf{c m}^{3}\right)$} & & \multicolumn{3}{c}{ Peso Fresco } \\
\hline DAA & L1 & L2 & L3 & L1 & L2 & L3 \\
180 & $0,00 \mathrm{bA}$ & $0,00 \mathrm{cA}$ & $0,00 \mathrm{bA}$ & $0,00 \mathrm{cA}$ & $0,00 \mathrm{cA}$ & $0,00 \mathrm{Ba}$ \\
225 & $1,38 \mathrm{aA}$ & $0,88 \mathrm{bB}$ & $0,47 \mathrm{aC}$ & $0,76 \mathrm{bA}$ & $0,49 \mathrm{bB}$ & $0,40 \mathrm{Ac}$ \\
275 & $1,25 \mathrm{aA}$ & $1,38 \mathrm{aA}$ & $0,44 \mathrm{aB}$ & $0,85 \mathrm{aA}$ & $0,71 \mathrm{aB}$ & $0,36 \mathrm{C}$ \\
\hline $\mathrm{Cv} \%$ & & & & & & 10,06 \\
\hline
\end{tabular}

DAA= Dia após a antese. $\mathrm{L}=$ Lote. $\mathrm{L} 1=$ Lote 1 L2 = Lote $2 ; \mathrm{L} 3=$ Lote 3 . Volume das sementes $\left(\mathrm{cm}^{3}\right)$; Peso fresco das sementes $(\mathrm{g})$. Médias seguidas pela mesma letra, minúscula na coluna ou maiúscula na linha, não diferem entre si pelo teste de Tukey, ao nível de 5\% de probabilidade.

Fonte: Autores.

A Tabela 7 retrata dos valores médios da germinação, primeira contagem, Índice de velocidade de germinação (IVG) e o peso de mil sementes aos 225 e 275 DAA. Dessa forma, foi possível observar que para a germinação, houve diferença estatística, e que o melhor percentual germinativo ocorreu no lote 2 (83 e 85\%) em ambos os períodos. A primeira contagem apresentou melhores médias no lote 1 e 2 (42 e 51\%) respectivamente aos 225 e 275 DAA, o lote 2 apresentou maior resultado (62\%), porém, não se diferenciou estatisticamente do lote 1.

Tabela 7. Fisiologia das sementes de cumbaru em diferentes épocas após antese.

\begin{tabular}{ccccc}
\hline & Germinação & Primeira contagem & IVG & Peso de mil \\
\hline L1 & $62 \mathrm{~b}$ & $42 \mathrm{a}$ & $2,35 \mathrm{a}$ & $787,14 \mathrm{a}$ \\
L2 & $83 \mathrm{a}$ & $51 \mathrm{a}$ & $2,87 \mathrm{a}$ & $503,50 \mathrm{~b}$ \\
L3 & $45 \mathrm{~b}$ & $23 \mathrm{~b}$ & $1,45 \mathrm{~b}$ & $404,88 \mathrm{c}$ \\
\hline L1 & & $\mathbf{2 7 5}$ DAA & $2,25 \mathrm{ab}$ & $862,81 \mathrm{a}$ \\
L2 & $64 \mathrm{~b}$ & $48 \mathrm{ab}$ & $2,75 \mathrm{a}$ & $730,48 \mathrm{~b}$ \\
L3 & $85 \mathrm{a}$ & $62 \mathrm{a}$ & $1,51 \mathrm{~b}$ & $355,41 \mathrm{c}$ \\
\hline CV 0 & $46 \mathrm{c}$ & $35 \mathrm{~b}$ & 20,91 & 7,49 \\
\hline
\end{tabular}

DAA= Dia após a antese. $\mathrm{L}=$ Lote. $\mathrm{L} 1=$ Lote1; L2 = Lote $2 ; \mathrm{L} 3=$ Lote 3. Germinação (\%); Primeira contagem (\%); IVG= Índice de velocidade de germinação; Peso de mil sementes $(\mathrm{g})$. Médias seguidas pela mesma letra, minúscula na coluna ou maiúscula na linha, não diferem entre si pelo teste de Tukey, ao nível de 5\% de probabilidade.

Fonte: Autores.

Para o IVG os lotes 1 e 2 em ambos os períodos apresentaram estatisticamente os melhores resultados. Em relação ao peso de mil sementes, as maiores médias foram observadas no lote 1 e 2 em ambos os períodos avaliados.

Mesmo com um teor de água ideal nas sementes, com exceção do lote 1 no $3^{\circ}$ período (Tabela 4), foi possível observar decréscimos na porcentagem de germinação e no IVG em sementes coletadas em no $3^{\circ}$ lote aos 275 dias após antese (Tabela 7). Essa menor germinação de plântulas no $3^{\circ}$ lote aos 275 dias após antese pode ser atribuída à perda da viabilidade das sementes, pela incidência de fungos (observados ao longo da instalação do experimento) ou pelo período de maturação incompleto. A primeira contagem apresentou melhores valores no lote 1 e 2 aos 225 e 275 DAA apenas no lote 2. 
Carvalho e Nakagawa (2000) descrevem que no período da maturação, as sementes crescem em tamanho até atingirem o valor característico para a espécie. Porém dentro da mesma espécie existem variações individuais devido à influência ambiental durante o desenvolvimento das sementes e da variabilidade genética entre as matrizes (Turnbull, 1975).

Para Gonçalves et al. (2013) as informações referentes à variação biométrica de parâmetros de frutos e sementes, é imprescindível para o melhoramento dessas características, seja no sentido de aumentar ou uniformizar, desta forma as variáveis estudadas podem ser utilizadas em programas de melhoramento.

\section{Conclusão}

Informações biométricas dos frutos e sementes do cumbaru podem ser relacionadas ao período de maturação dos mesmos.

Os frutos e sementes de cumbaru apresentam boa qualidade morfológica a partir do $2^{\circ}$ período de coleta (225 DAA).

A melhor qualidade fisiológica das sementes é encontrada entre os 225 e 275 DAA, recomendando a colheita dos frutos nesse período.

Contudo, sugere-se para trabalhos futuros a adição de outros intervalos de períodos de coletas de frutos de Dipteryx alata. Vog.

\section{Referências}

Aguiar, F. F. A., Tavares, A. R., Kanashiro, S., Luz, P. B. D., \& Santos Júnior, N. A. D. (2010). Germinação de sementes de Dalbergia nigra (Vell.) Allemao ex Benth. (Fabaceae-Papilionoideae) no armazenamento. Ciência e Agrotecnologia, 34(SPE), 1624-1629.

Alvarenga, C. R., Jorge, M. H. A. Cumbaru no Pantanal. Corumbá, MS: Embrapa Pantanal, 2008. 2p. ADM - Artigo de Divulgação na Mídia, n.127. <http://www.cpap.embrapa.br/publicacoes/online/ADM127>.

Arakaki, A. H., Scheidt, G. N., Portella, A. C., Arruda, E. J. D., \& Costa, R. B. D. (2009). O baru (Dipteryx alata Vog.) como alternativa de sustentabilidade em área de fragmento florestal do Cerrado, no Mato Grosso do Sul. Interações (Campo Grande), 10(1), 31-39.

Ataíde, G. D. M., Gonçalves, J. F. D. C., Guimarães, V. M., Flores, A. V., \& Bicalho, E. M. (2013). Alterations in seed reserves of Dalbergia nigra ((Vell.) Fr All. ex Benth.) during hydration. Journal of Seed Science, 35 (1), 56-63.

Basso, S. M. S. (1999). Caracterização morfológica e fixação biológica de nitrogênio de espécies de Adesmia DC. e Lotus L. Tese de doutorado em Zootecnia, Universidade Federal do Rio Grande do Sul, RS, Brasil.

Botezelli, L., Davide, A. C., \& Malavasi, M. M. (2000). Características dos frutos e sementes de quatro procedências de Dipteryx alata Vogel (Baru). Cerne, 6(1), 9-18.

Carvalho, N. M., \& Nakagawa, J. (2000). Sementes ciência, tecnologia e produção: Funesp.

Felippi, M., Araújo, M. M., Longhi, S. J., \& Lucio, A. D. (2015). Fenologia reprodutiva e qualidade das sementes de Cabralea canjerana (Vell.) Mart. Ciência Rural, 45(12), 2137-2142.

Felippi, M., Maffra, C. R. B., Cantarelli, E. B., Araújo, M. M., \& Longhi, S. J. (2012). Fenologia, morfologia e análise de sementes de Apuleia leiocarpa (Vogel) JF Macbr. Ciência Florestal, 22(3), 477-491.

Ferreira, D. F. (2014). Sisvar: a Guide for its Bootstrap procedures in multiple comparisons. Ciência e agrotecnologia, 38(2), 109-112.

Ganga, R. M. D., Chaves, L. J., \& Naves, R. V. (2009). Parâmetros genéticos em progênies de Hancornia speciosa Gomes do Cerrado. Scientia Forestalis, 37(84), 395-404.

Gomes, K. B. P., Martins, R. D. C. C., Martins, I. S., \& Gomes Junior, F. G. (2014). Avaliação da morfologia interna de sementes de Terminalia argentea (Combretaceae) pelo teste de raios X. Revista Ciência Agronômica, 45(4), 752-759.

Gonçalves, L. G. V., Andrade, F. R., Marimon Junior, B. H., Schossler, T. R., Lenza, E., \& Marimon, B. S. (2013). Biometria de frutos e sementes de mangaba (Hancornia speciosa Gomes) em vegetação natural na região leste de Mato Grosso, Brasil. Revista de Ciências Agrárias, 36(1), 31-40.

Guimarães, R. A., Miranda, K. M. C., Mota, E. E. S., Chaves, L. J., Telles, M. P. D. C., \& Soares, T. N. (2019). Avaliação da diversidade genética e estrutura populacional em uma coleção de germoplasma de Dipteryx alata utilizando marcadores microssatélites. Crop Breeding and Applied Biotechnology, 19 (3), 329-336.

Gusmão, E., Almeida Vieira, F., \& da Fonseca Júnior, É. M. (2006). Biometria de frutos e endocarpos de murici (Byrsonima verbascifolia Rich. ex A. Juss.). Cerne, 12(1), 84-91. 
Research, Society and Development, v. 10, n. 1, e21610111589, 2021

(CC BY 4.0) | ISSN 2525-3409 | DOI: http://dx.doi.org/10.33448/rsd-v10i1.11589

Hehenberger, E., Kradolfer, D., \& Köhler, C. (2012). Endosperm cellularization defines an important developmental transition for embryo development. Development, 139(11), 2031-2039.

Instituto Brasileiro de Geografia e Estatística (IBGE). (2017). Censo de 2015. 〈http:///www.sidra.ibge.gov.br/bda/agric/〉.

Lorenzi, H. (1998). Árvores brasileiras - manual de identificação e cultivo de plantas arbóreas do Brasil (No. C/582.1609 L6). Plantarum.

Luz, K. C. (2016). Variação genética entre e dentro de populações de Dipteryx alata vog. para caracteres morformétricos de plântulas, frutos e sementes. Dissertação de mestrado em Sistemas de Produção, Faculdade de Engenharia de Ilha Solteira, SP, Brasil.

Maguire, J. D. (1962). Speeds of germination-aid selection and evaluation for seedling emergence and vigor. Crop Science. 2(2), 176-177.

Ministério da Agricultura, Pecuária e Abastecimento. Secretaria de Defesa Agropecuária. (2009). Regras para análise de sementes.

Marcos Filho, J. M. F. (2005). Fisiologia de sementes de plantas cultivadas. Fealq.

Marcos-Filho, J. (2015). Fisiologia de sementes de plantas cultivadas: ABRATES.

Neves, S. M. A. S., Nunes, M. C. M., \& Neves, R. J. (2011). Caracterização das condições climáticas de Cáceres/MT-Brasil, no período de 1971 a 2009: subsídio às atividades agropecuárias e turísticas municipais. Boletim Goiano de Geografia, 31(2), 55-68.

Oliveira, M. I. B., \& Sigrist, M. R. (2008). Fenologia reprodutiva, polinização e reprodução de Dipteryx alata Vogel (Leguminosae-Papilionoideae) em Mato Grosso do Sul, Brasil. Brazilian Journal of Botany, 31(2), 195-207.

Oliveira-Alves, S. C., Pereira, R. S., Pereira, A. B., Ferreira, A., Mecha, E., Silva, A. B., \& Bronze, M. R. (2020). Identificação de compostos funcionais em nozes de baru (Dipteryx alata Vog.): Valor nutricional, composição volátil e fenólica, atividade antioxidante e efeito antiproliferativo. Food Research International, 131, 109026.

Pinho, E. K. C., Lopes, A. N. K., Costa, A. C., Silva, A. B. V., Vilar, F. C. M., Reis, R. G. E. Substratos e tamanhos de pool na produção de mudas de baruzeiro (Dipteryx alata Vog.). Ciência Agrícola, Rio Largo,16(1),11-19, 2018.

Sano, S. M., Ribeiro, J. F., \& Brito, M. A. (2004). Baru: biologia e uso. Embrapa Cerrados-Documentos (INFOTECA-E).

Sano, S. M., Ribeiro, J. F., \& Brito, M. A. (2014). Baru: biologia e uso. Ministério da Agricultura, Pecuária e Abastacimento -MAPA. EMBRAPA.

Santos, C. L., Junior, S. S., de Lalla, J. G., Theodoro, V. C. A., \& Nespoli, A. (2009). Desempenho de cultivares de alface tipo crespa sob altas temperaturas em Cáceres-MT. Agrarian, 2(3), 87-98.

Silva Junior, V. T. D., Lima, J. M. G. M. D., Rodrigues, C. W. D. M. S., \& Barbosa, D. C. D. A. (2012). Erythrina velutina Willd. (LeguminosaePapilionoideae) ocorrente em caatinga e brejo de altitude de Pernambuco: biometria, embebição e germinação. Revista Árvore, 36(2), $247-257$.

Silva, G. P., Vendas, J. F., Nascimento, K. J. T., Rodrigues, A. A., Camelo, G. N., \& Borges, E. E. D. L. (2020). Alterações bioquímicas e fisiológicas em Dipteryx alata Vog. sementes durante a germinação e envelhecimento acelerado. South African Journal of Botany, 131, 84-92.

Silva, G. P. (2017). Alterações fisiológicas e bioquímicas de sementes de Dipteryx alata vog. No processo de germinação e armazenamento. Rio Verde, Tese (Doutorado em Ciências agrárias)

Silva, L. J. D., Dias, D. C. F. D. S., Sekita, M. C., \& Finger, F. L. (2018). Lipid peroxidation and antioxidant enzymes of Jatropha curcas L. seeds stored at different maturity stages. Acta Scientiarum. Agronomy, 40(1), 34978.

Soares, T. N., Chaves, L. J., Telles, M. P. D. C., Diniz-Filho, J. A. F., \& Resende, L. V. (2008). Distribuição espacial da variabilidade genética intrapopulacional de Dipteryx alata. Pesquisa Agropecuária Brasileira, 43(9), 1151-1158.

Souza, R. G. M., Gomes, A. C., Castro, I. A., Mota, J. F. (2018). Uma dieta enriquecida com amêndoas de baru reduz a adiposidade abdominal e melhora as concentrações de lipoproteínas de alta densidade: um ensaio randomizado controlado por placebo. Nutrition, 55, 154-160.

Turnbull, J. W. (1975). Seed extraction and cleaning. In FAO/DANIDA Training Course on Forest Seed Collection and Handling. Chiang Mai (Thailand), $135-151$.

Zuffo, A. M., Andrade, F. R., \& Zuffo Júnior, J. M. (2014). Caracterização biométrica de frutos e sementes de baru (Dipteryx alata Vog.) na região leste de Mato Grosso, Brasil. Revista de Ciências Agrárias, 37(4), 463-471. 